\title{
Effectiveness of Visual-Tactile Examination and DIAGNOdent Pen in Detecting Early Enamel Caries and Its Remineralisation: An In Vitro Study
}

\author{
Mohammed Fadhil Rashid, ${ }^{1}$ Mohmed Isaqali Karobari $\mathbb{D}^{1,1,2,3}$ \\ Mohamad Syahrizal Halim $\mathbb{D}^{1,4}$ and Tahir Yusuf Noorani ${ }^{1,4}$ \\ ${ }^{1}$ Conservative Dentistry Unit, School of Dental Sciences, Universiti Sains Malaysia, Health Campus, Kubang Kerian, Kota Bharu, \\ 16150 Kelantan, Malaysia \\ ${ }^{2}$ Department of Restorative Dentistry \& Endodontics, Faculty of Dentistry, University of Puthisastra, Phnom Penh 12211, Cambodia \\ ${ }^{3}$ Center for Transdisciplinary Research (CFTR), Saveetha Dental College, Saveetha Institute of Medical and Technical Sciences, \\ Saveetha University, Chennai, Tamil Nadu 600077, India \\ ${ }^{4}$ Conservative Dentistry Unit, Hospital Universiti Sains Malaysia, Health Campus, Kubang Kerian, Kota Bharu 16150, \\ Kelantan, Malaysia
}

Correspondence should be addressed to Mohmed Isaqali Karobari; dr.isaq@gmail.com and Tahir Yusuf Noorani; dentaltahir@yahoo.com

Received 29 October 2021; Revised 16 December 2021; Accepted 17 December 2021; Published 11 January 2022

Academic Editor: Iole Vozza

Copyright (c) 2022 Mohammed Fadhil Rashid et al. This is an open access article distributed under the Creative Commons Attribution License, which permits unrestricted use, distribution, and reproduction in any medium, provided the original work is properly cited.

Background. The caries preventive effect of Colgate Duraphat ${ }^{\circledR}$ and GC Tooth Mousse Plus ${ }^{\circledR}$ has been widely studied, but the remineralisation potential of initial occlusal caries using these two remineralisation materials remains unclear. Aim. This study is aimed at evaluating and comparing the remineralisation of early enamel caries on the occlusal surface of permanent posterior teeth using ICDAS II caries scoring system and DIAGNOdent Pen (DDPen) after remineralisation with Colgate Duraphat ${ }^{\circledR}$ and GC Tooth Mousse Plus ${ }^{\circledR}$. Materials and Methods. Extracted posterior teeth $(N=120)$ with incipient occlusal caries were included in this study. The occlusal surface of each tooth was scored using DDPen and ICDAS II scoring before remineralisation. Then, remineralisation of the teeth of the experimental group was carried out using either CPP-ACP-F or fluoride varnish. After the remineralisation procedures, the occlusal surface of each tooth was again scored using DDPen and ICDAS II scoring. The teeth were then fixed in dental stone blocks and sectioned longitudinally for histological examination using a stereomicroscope. Statistical analysis was performed to calculate the sensitivity and specificity of DDPen and ICDAS II to detect remineralisation and compare with the gold standard histological examination. Results. According to ICDAS-II scores, a significant difference was noted in GC Tooth Mousse Plus ${ }^{\circledR}$ and Duraphat ${ }^{\circledR}$ study samples, whereas the difference between the pre-and post-remineralisation of the control group was not significant. According to the DDPen score criteria, a statistically significant difference was noted among all study groups; however, a greater significance level was noted in the GC Tooth Mousse Plus ${ }^{\circledR}$ and Duraphat ${ }^{\circledR}$ study samples compared with the control group. The Spearman's rank correlation of ICDAS-II and DDPen with Downer's histological score (gold standard) revealed a higher association of DDPen score (.738) as compared to ICDAS-II scores (.430). Conclusion. The study concluded that both ICDAS II and DDPen could detect remineralisation of early enamel occlusal caries. DDPen was more sensitive than ICDAS-II to detect remineralisation compared with the Downers histological scores. 


\section{Introduction}

Dental caries is a gradually progressive disease that has been identified as the most commonly occurring oral disease/ chronic infection $[1,2]$. The diagnosis of a noncavitated carious lesion is considered challenging because this lesion can be disguised by the remineralisation effect of fluorides [3]. Visuo-tactile inspection of the carious lesion is an inexpensive and the most utilised method of caries assessment [4]. The accuracy and reliability of a diagnosis depend on the expertise and training of the assessor. A standardised caries assessment system is of paramount importance [5].

Several studies have reported that Duraphat ${ }^{\circledR}$ is highly effective in remineralising enamel from other mineralisation products [6-9]. Further research has found that a high concentration of fluoride treatments in acidic $\mathrm{pH}$ is more effective in reshaping the surface of noncavitated caries and encouraging fluoride absorption from those in neutral $\mathrm{pH}$ [10]. Casein phosphopeptide-amorphous calcium phosphate has been highlighted to enhance the stability of high calcium levels and enhance the delivery of ions to the tooth surface $[11,12]$. A clinical trial involving CPP-ACP containing gum demonstrated decelerated progression and enhanced regression of carious lesions [13]. CPP-ACP application also showed postorthodontic white spot regression [14]. Furthermore, CPP-ACP has a significant remineralisation effect, and it can be considered a salivary biomimetic, as it shares many similarities with statherin [15].

ICDAS II supplies a consistent method of lesion detection and evaluation, leading to the diagnosis of caries [16]. ICDAS II provides good reproducibility and accurate detection in vivo and in vitro for initial caries lesions at different stages. The laser fluorescence method (DIAGNOdent) assists the detection of occlusal and approximal caries [2]. The use of the DDPen on white spot carious lesions [17] and an enamel window at the buccal surface in the middle one-third of the crown [18] has been reported. However, the use of DDPen to assess the remineralisation of the fissures, pits, and smooth surfaces has not been explored. Previous studies employed a relatively smaller sample size in their studies $[19,20]$. Varying cut-off values have been used and recommended by previous studies [21, 22]. However, these values are dependent on various factors which include type of surface (smooth or fissure), physical properties (demineralisation or remineralisation), and the extent of the lesion (lesion confined to enamel only and lesion involving enamel and dentin).

Narrative and systematic reviews have shown that DDpen is more sensitive to the occlusal aspect of posterior teeth than traditional diagnostic methods, but the specificity is inferior to clinical visual examination [23-25]. DDPen also tends to overestimate deeper caries or dark (stained) lesions. Bhat et al. [26] conducted a systematic review assessing different cut-off values and concluded that the cut-off values based on the laboratory studies were not clinically correlated. The study suggested that the cut-off values may be selected based on the extent of the carious lesion, and different cut-off values were correlated with enamel and dentinal carious lesions.
Different cut-off values for DDPen have been described in the literature; however, no study has evaluated pre-and post-remineralisation relevance of cut-off values. Moreover, no previous studies have been carried out performing simultaneous comparison between the three assessment tools (ICDAS II, DDPen, and histological examination) on tooth surface remineralisation. Hence, this study is aimed at investigating the potential of ICDAS II and DDPen in detecting remineralisation of the early carious lesion after applying topical fluoride gel (Colgate Duraphat ${ }^{\circledR}$ ) and casein phosphopeptide-amorphous calcium phosphate fluoride (Tooth Mouse Plus) at the occlusal surface of extracted human permanent posterior teeth. This study also compared the diagnostic cut-off values of DDPen corresponding with the histological examination after the remineralisation process.

\section{Materials and Methods}

Ethical approval for the current study was obtained from the Human Research Ethics Committee of Universiti Sains Malaysia (USM) (USM/JEPeM/18100516). Extracted permanent maxillary and mandibular posterior teeth for orthodontic, periodontal, or other reasons from adult patients that visited the outpatient dental clinics of the School of Dental Sciences, USM, were selected for this study.

A total of 120 freshly extracted teeth were included in this study. Before caries assessment, the occlusal surfaces were cleaned using a toothbrush with pumice slurry and water before randomly assigning numbers from 1 to 120 . Unrestored mandibular or maxillary posterior teeth without any carious lesion or incipient carious lesion limited to the enamel, denoted as code 01,02 , and 03 according to the ICDAS II scoring system, were included. The teeth samples were examined under $10 \mathrm{x}$ magnification individually. Teeth were excluded from the study if there was the presence of dental fluorosis, tetracycline staining or any sources of staining, hypoplasia, and dentinal exposure.

Before the remineralisation process, two trained and calibrated examiners (T.Y.N and M.S.H) performed the visual assessment of each sample using ICDAS II. Visual examination was using a dental operating light, a WHO probe, and a 3-way syringe to score each tooth according to the ICDAS II scoring system. Sharp explorers were not used during the visual diagnosis. TYN and MSH scored the teeth individually with no discussion regarding the scores to ensure blinding. Interexaminer reproducibility was assessed using kappa analysis. The third examiner (M.F.R) used the DDPen to score each sample afterwards. A triple air syringe was used for five seconds to dry out the enamel surface before using the DDPen. Before scoring, the device was calibrated according to manufacturer's instructions. The probe tip was positioned on the occlusal surface of the tooth and rotated around its vertical axis until the highest value was found. ICDAS II scores and DDPen readings were repeated for two weeks for all samples to ascertain and evaluate intraexaminer reproducibility.

For the control group, the extracted teeth samples were washed with deionised water and then placed in artificial 
saliva at $37^{\circ} \mathrm{C}$. No material was used for remineralisation of the control group. For the CPP-ACP with fluoride (GC Tooth Mousse Plus ${ }^{\circledR}$ ) group, the paste was applied once daily (every $24 \mathrm{~h}$ ) for 21 days on the occlusal surfaces with a microbrush tip applicator left in place for three minutes which simulated at-home use of this cream. The specimens were then rinsed with deionised water and gently dried with an air syringe. The samples were then immersed in artificial saliva and incubated at $37^{\circ} \mathrm{C}$ [26]. This process was repeated for 21 days. For the fluoride varnish (Colgate Duraphat ${ }^{\circledR}$ ) group, a thin layer of the Duraphat ${ }^{\circledR}$ was applied once using a microbrush tip applicator on tooth's enamel surface, which simulated the professional application in a dental visit. The samples were then immersed in artificial saliva at $37^{\circ} \mathrm{C}$ for $6 \mathrm{~h}$ [27] to simulate the oral environment. Then, the varnish was carefully removed using a toothbrush $[27,28]$ to ensure the complete elimination of the surface layer of varnish $[6$, 29]. The samples were then rinsed for one minute with deionised water. After that, the samples were immersed in artificial saliva and incubated at $37^{\circ} \mathrm{C}$.

After the remineralisation process, MFR repeats the scoring using the DDPen post-remineralisation for each sample. To ensure blinding, the examiner did not have access to previous scores of ICDAS or DDPen, and the same procedure for performing the post-remineralisation ICDAS score was followed. The teeth were then fixed in dental stone blocks and sectioned longitudinally using a diamond-coated bandsaw (Exakt system) for histological examination using a stereomicroscope with 10x magnification. Parallel cutting of the samples was performed by using tweezer prongs. The parallelism of the block is of utmost importance to ensure accurate cutting direction. The teeth sections fixed in the blocks were then polished by using slurry pumice. The polished sections were then again examined at 10x magnification under the stereomicroscope. Each section was photographed using a digital camera. This gold standard histological examination was compared with the ICDAS II scoring and DDPen examination for validity assessment.

The descriptive statistical analysis was performed using SPSS software version 24 (IBM SPSS Statistics). Inter- and intraexaminer reproducibility was assessed by calculating the unweighted kappa coefficient [30]. Wilcoxon signed ranks for pre-and post-remineralisation using different caries diagnostic tests were performed to assess the potential to detect remineralisation. Spearman's rank correlation of ICDAS-II and DDPen with the histological gold standard was performed to check the association. Sensitivity, specificity, and area under the ROC were calculated for ICDAS II and DDPen scores to compare both caries diagnostic methods.

\section{Results}

Kappa statistics revealed almost perfect reproducibility of TYN (0.954), MSH (0.919), and MFR (0.885), whereas good interexaminer agreement (0.673) was observed [31]. Table 1 describes the difference between the pre- and post-remineralisation scores, which were assessed using ICDAS-II and DDPen. According to ICDAS-II scores, a significant difference was noted in GC Tooth Mousse Plus ${ }^{\circledR}$
TABLE 1: Wilcoxon signed ranks for pre- and post-remineralisation using different caries diagnostic tests.

\begin{tabular}{|c|c|c|}
\hline & $Z$ & $p$ value \\
\hline \multicolumn{3}{|l|}{ ICDAS-II } \\
\hline Control & -1.342 & .180 \\
\hline Tooth Mousse Plus ${ }^{\circledR}$ & -3.285 & $.001^{* *}$ \\
\hline Duraphat $^{\circledR}$ & -2.592 & $.010^{*}$ \\
\hline \multicolumn{3}{|l|}{ DDPen score } \\
\hline Control & -2.928 & $.003^{* *}$ \\
\hline Tooth Mousse Plus ${ }^{\circledR}$ & -4.379 & $<.001^{* *}$ \\
\hline Duraphat ${ }^{\circledR}$ & -4.411 & $<.001^{* *}$ \\
\hline
\end{tabular}

and Duraphat ${ }^{\circledR}$ study samples, whereas the difference between the pre- and post-remineralisation of the control group was not significant. According to the DDPen score criteria, a statistically significant difference was noted among all study groups; however, a greater significance level was noted in the GC Tooth Mousse Plus ${ }^{\circledR}$ and Duraphat ${ }^{\circledR}$ study samples compared with the control group. Table 2 presents kappa values of the DDPen score with different cut-off values. The DDPen was noted to have the highest agreement compared with the histological gold standard described by Lussi et al. [32]. Table 3 presents the frequency of true-positive and false-positive findings of the carious occlusal lesion. The Spearman's rank correlation of ICDAS-II and DDPen with the histological gold standard revealed a higher association of DDPen score (.738) than ICDAS-II scores (.430).

Figure 1(a) presents the ROC curves plotted for the ICDAS-II code 0 and DDPen score (0-13) measurements compared with Downer's histological level DI extent as a gold standard validation. Table 4 presents the $A z$ value of DDPen score (0-13) at D1 (0.972) was higher than ICDASII code 0 at D1 (0.811), showing the greater accuracy of the method. Rank correlations (Spearman's coefficient) with histology D1 were .692 for ICDAS-II code 0 and .950 for DDPen score (0-13). Figure 2(a) represents the histological section of the tooth at Downer's classification level D1.

Figure 1(b) presents the ROC curves plotted for the ICDAS-II codes 1 and 2 and DDPen score (14-20) measurements using Downer's histological level D2 extent as a gold standard validation. Table 4 presents the $A z$ value of DDPen score (14-20) at D2 (0.894) was higher than ICDAS-II codes 1 and 2 at D2 (0.667), showing the greater accuracy of the method. Rank correlations (Spearman's coefficient) with histology D2 were .769 for ICDAS-II codes 1 and 2 and .854 for DDPen score (14-20). Figure 2(b) represents the histological section of a tooth at Downer's classification level D2.

Figure 1(c) presents the ROC curves plotted for the ICDAS-II code 3 and DDPen score $(>21)$ measurements using Downer's histological level D3 extent as a gold standard validation. Table 4 presents the $A z$ value of DDPen score $(>21)$ at D3 (0.838) was higher than ICDAS-II code 3 at D3 (0.721), showing the greater accuracy of the method. Rank correlations (Spearman's coefficient) with histology D3 were .601 for ICDAS-II code 3 and .774 for DDPen score 
TABLE 2: Kappa statistics for different cut-off values of DDPen score.

\begin{tabular}{lccccc}
\hline DDPen criteria & Sound & \multicolumn{2}{c}{$\begin{array}{c}\text { Histological gold standard } \\
\text { Demineralised enamel } \\
\text { Inner half }\end{array}$} & Demineralised dentine & Kappa value \\
\hline$[32]$ & $0-13$ & $14-20$ & & $>21$ & $.725^{* *}$ \\
{$[44]$} & $0-14$ & $15-21$ & & $>25$ & .627 \\
{$[35]$} & $0-15$ & $16-25$ & $11-18$ & $>18$ & .437 \\
{$[33]$} & $0-4$ & $5-10$ & & & .457 \\
\hline
\end{tabular}

TABle 3: Percentage of occlusal carious lesions detected correctly utilizing different caries diagnostic methods at different histological levels.

\begin{tabular}{|c|c|c|c|c|}
\hline \multirow[t]{2}{*}{ Caries diagnostic method } & \multirow[t]{2}{*}{ Histology score } & \multicolumn{2}{|c|}{$\begin{array}{c}\text { Percentage of occlusal carious lesions detected } \\
\text { correctly at different histological levels } n \%\end{array}$} & \multirow[t]{2}{*}{$r_{S}(\mathrm{SE})$} \\
\hline & & True positive-false positive/total & $\%$ & \\
\hline \multicolumn{5}{|l|}{ ICDAS-II } \\
\hline Sound (code 0 ) & D1 & $33-20 / 53$ & 62.26 & \\
\hline ICDAS (codes 1 and 2) & D2 & $11-22 / 33$ & 33 & $.430(.084)$ \\
\hline ICDAS (code 3) & D3 & $15-19 / 34$ & 44.117 & \\
\hline \multicolumn{5}{|l|}{ DDPen score } \\
\hline $0-13$ & D1 & $50-3 / 53$ & 94.33 & \\
\hline $14-20$ & $\mathrm{D} 2$ & $26-7 / 33$ & 78.78 & $.738(.062)$ \\
\hline$>21$ & D3 & $23-11 / 34$ & 67.64 & \\
\hline
\end{tabular}

\%: percentage; $r_{s}$ : Spearman rank correlation; $D$ : Downer's classification.

$(>21)$. Figure 2(c) represents the histological section of a tooth at Downer's classification level D3.

\section{Discussion}

The current study is aimed at evaluating the sensitivity and specificity of ICDAS II and DDPen to detect the remineralisation of early enamel caries lesion in extracted human permanent posterior teeth and compare with the gold standard histological examination to identify the most suitable cut-off values of DDPen for identification of remineralised carious lesions.

The current study utilised ICDAS-II and DDPen scoring, which are designed to enable the clinicians to diagnose the disease at the chairside, making the results of the current study more relatable to a clinical setup. The current study did not find a significant difference in the remineralisation potential of GC Tooth Mousse Plus ${ }^{\circledR}$ and Duraphat ${ }^{\circledR}$, following previous literature [6]. However, the effect of GC Tooth Mousse Plus ${ }^{\circledR}$ on salivary constituents and $\mathrm{pH}$ value were not considered. Varying cut-off limits for DDPen have been proposed and adopted in the laboratory $[33,34]$ and clinical studies [32, 35, 36]. Heinrich-Weltzien et al. [37] conducted a systematic review assessing different cut-off values and concluded that the cut-off values might be selected based on the extent of the carious lesion, and different cut-off values were correlated with enamel and dentinal carious lesions.

The cut-off values for DDPen, which had the highest and a significant correlation $\left(0.72^{*}\right)$ with Downer's histological scores for the corresponding study samples, agree with the cut-off value suggested by Lussi et al. [32]. A possible expla- nation of this close agreement could be the assessment of freshly extracted teeth and the preservation medium (artificial saliva). The use of storage solutions such as formalin, sodium azide, or thymol-based mediums has been reported to affect the DDPen measurements $[34,38]$. The values of fluorescence emission intensity decrease with time of immersion in all storage solutions, except for artificial saliva and glutaraldehyde [39]. The storage solutions tend to remove the organic compounds from the enamel surface. It is important to note that distilled water does not remove the organic compounds after 30 days of immersion [39]. Immersion in $0.1 \%$ thymol solution has shown a decline compared to the laser fluorescence values [40]. The area under the ROC curve (AUROC) can be defined as a composite measure of accuracy. Metz [41] stated that AUROC is a more meaningful measure of the value of a diagnostic test than the overall accuracy, as it does not depend on the disease prevalence in the population. The "diagnostic test" in the current study, being either ICDAS-II or DDPen, is compared with the gold standard Downers histological classification and "the disease" being remineralisation of the carious lesion.

An advantage of the area under the ROC curve analysis is that it reflects the diagnostic performance more comprehensively than the sensitivity and specificity, determined by only one cut-off point. It also provides an overall validity of the methods [42, 43]. Diniz et al. [44] performed an in vivo evaluation of the DDPen utilising the area under the ROC curve and reported that the $A z$ value at D1 (0.72), D2 (0.81), and D3 (0.93), whereas another clinical study by Heinrich-Weltzien et al. [37] reported higher $A z$ 


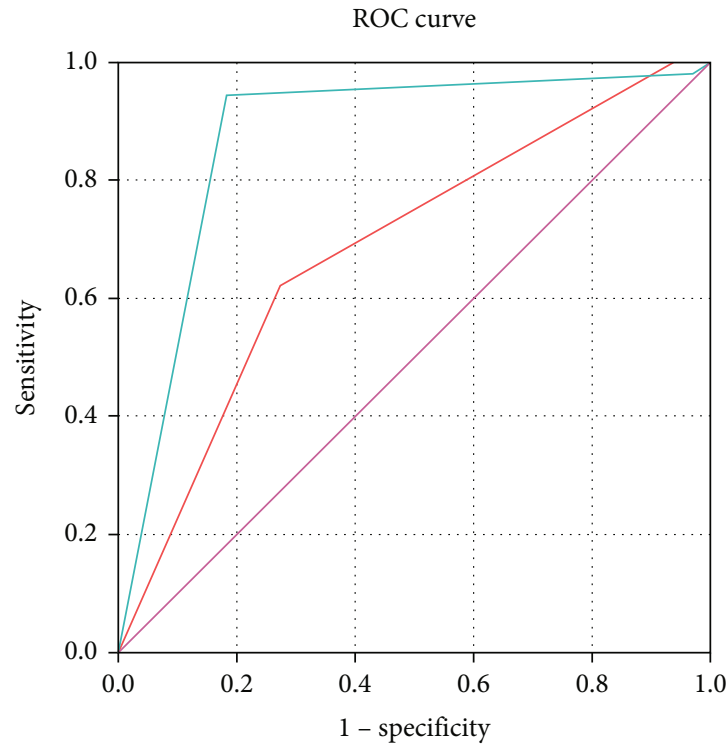

Diagonal segments are produced by ties.

Source of the curve

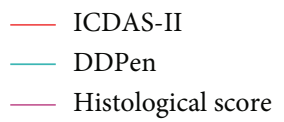

(a)

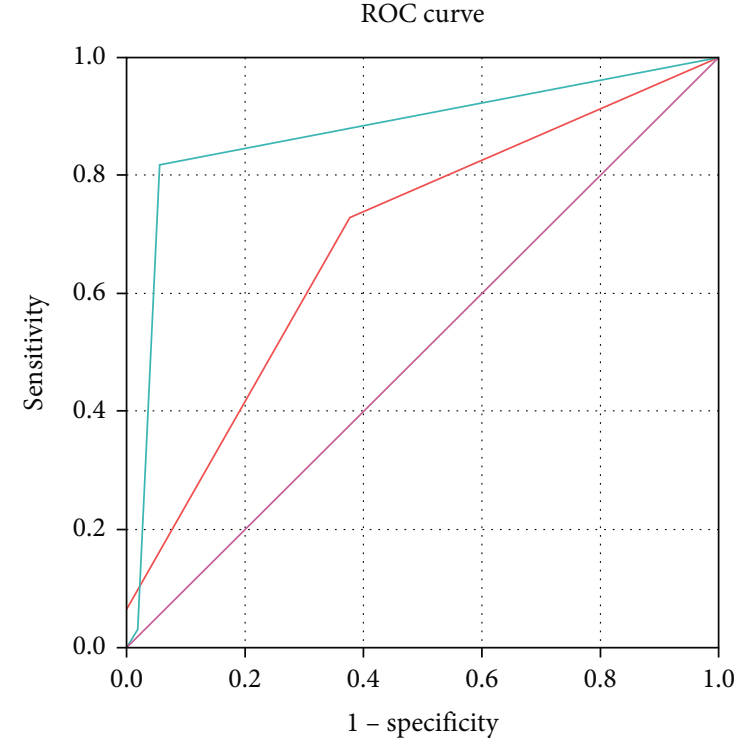

Diagonal segments are produced by ties.

Source of the curve

$$
\begin{aligned}
& \text { — ICDAS-II } \\
& - \text { DDPen } \\
& \text { _ Histological score }
\end{aligned}
$$

(b)

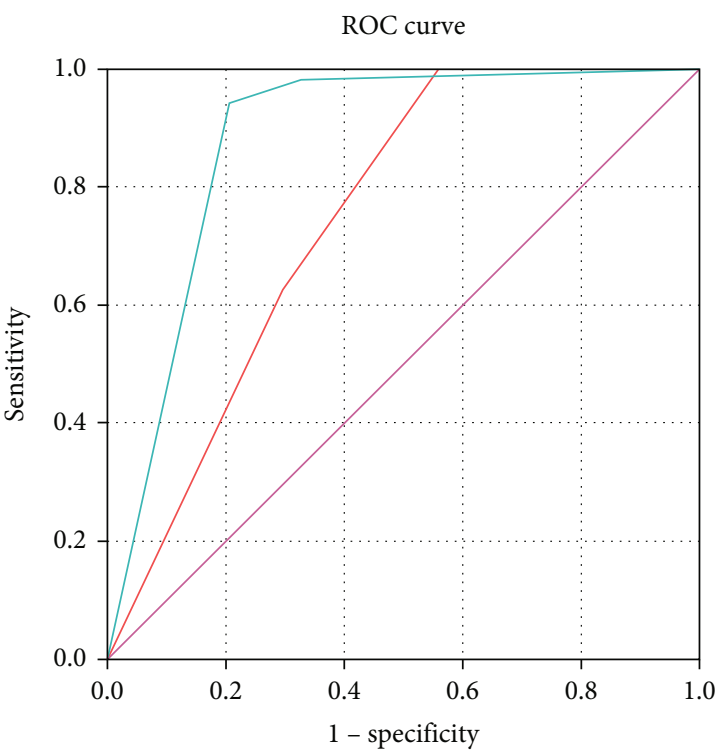

Diagonal segments are produced by ties.

Source of the curve

ICDAS-II
ICDPen
_ Histological score

(c)

Figure 1: Area under the receiver-operating characteristic (AUROC) for International Caries Detection and Assessment System (ICDAS) codes under binary groups correlated with Downer's histologic classification at (a) D1 threshold, (b) D2 threshold, and (c) D3 threshold. 
TABLE 4: Spearman's correlation coefficients, AUROC curve, specificity, and sensitivity for the mode ICDAS scores, DDPen when compared with the level D1, D2, and D3 Downer's histologic level.

\begin{tabular}{lccccccc}
\hline & Histo score & $r_{s}$ & Standard error & $A_{z}(95 \%$ CI $)$ & Standard error & Sensitivity & Specificity \\
\hline ICDAS II code 0 & D1 & .692 & .053 & $.811(.726-.897)$ & .044 & .623 & .284 \\
DDPen cutoff 0-13 & D1 & .950 & .028 & $.972(.935-1.000)$ & .034 & .943 \\
ICDAS II codes 1 and 2 & D2 & .769 & .059 & $.667(.557-.776)$ & .056 & .667 & .333 \\
DDPen cutoff 14-20 & D2 & .854 & .050 & $.894(.810-.978)$ & .043 & .788 & .069 \\
ICDAS II code 3 & D3 & .601 & .067 & $.721(.605-.837)$ & .059 & .441 \\
DDPen cutoff $>21$ & D3 & .774 & .058 & $.838(.740-.937)$ & .050 & .676 \\
\hline
\end{tabular}

$A_{z}$ : area under receiver-operator curve; CI: confidence interval; $D$ : Downer's classification; $r_{s}$ : Spearman's coefficient; $S_{n}$ : sensitivity; $S_{P}$ : specificity.

values for D2 (0.90) and D3 (0.83). The current study reported the highest $\mathrm{Az}$ value of DDPen score (0-13) at D1 (0.972) followed by DDPen score (14-20) at D2 (0.894) and then DDPen score $(>21)$ at D3 (0.838). The difference in the performance of DDPen can be attributed to the determination of cut-off values or the number of cases reported in each study. Our study included the most significant number of cases having carious lesions at the D1 level, which could better represent higher accuracy in that category.

Iranzo-Cortés et al. [45] conducted a study focusing on occlusal caries by comparing the performance of ICDAS II and DDPen. The study concluded that the adjunct use of DDPen for the diagnosis of carious lesions was advisable. The results showed that DDPen demonstrated higher sensitivity, and ICDAS-II demonstrated higher specificity, following the current study results. A balance between sensitivity and specificity of a diagnostic tool is desired to ensure correct diagnosis and effective treatment planning. The current study found higher sensitivity of DDPen, which can sometimes lead to excessive treatment, which might not be deemed necessary when evaluated clinically.

On the other hand, a higher specificity is desirable to ensure minimal false-positive cases, which was found true in the case of ICDAS II. A possible explanation of this finding is the subjective nature of ICDAS criteria, which provides clinicians with a specific demarcation of disease progression. Similarly, Akgul et al. [1] performed a diagnostic evaluation of incipient carious lesion using visual inspection (VI), DDPen, DIAGNOdent Camera, and alternatingcurrent-impedance-spectroscopy technique (ACIST). The study concluded that either of the diagnostic devices, when used alone, are not sensitive enough to diagnose incipient carious lesions, and these devices must be used as an adjunct to the traditional visual inspection method. The current study results also warrant the use of DDPen as an adjunct to the ICDAS II scoring system as both methods have their benefits, which add up together.

The spearman rank correlation coefficient was calculated for different lesion depths based on Downer's histological classification. The rank score of DDPen ranged from 0.774 to 0.950 compared to the ICDAS-II rank score ranging from 0.601 to 0.769 . However, these results revealed the potential of ICDAS-II and DDPen to detect remineralisation. Another notable finding is that the most significant difficulty in diagnosing carious lesions was observed in deeper carious lesions with the lowest accuracy. The results revealed that DDPen could be utilised as a valuable clinical tool to detect remineralisation and monitor the progress of remineralisation of a particular lesion.

Alomari et al. [46] carried out a laboratory study evaluating the effectiveness by measuring the sensitivity and specificity of visual-only, visual + radiography, visual + radiography + DDPen for diagnosing noncavitated occlusodentinal caries. The study concluded that although the use of radiography and DDPen adjunctively had a benefit, the $A z$ value did not present a statistically significant difference, meaning that the diagnosis and decision-making were not influenced in the three study groups. In comparison, our study focused on carious lesions and found that DDPen was significantly (rank correlation $=0.738$ ) more effective in diagnosing cavitated carious lesions when compared with ICDAS-II (rank correlation $=0.430$ ), which warrants the use of DDPen in cavitated lesions as an adjunct. The higher accuracy of DDPen and electronic caries monitor has also been previously reported [47]. A possible explanation of this difference might be the colour of the lesion, which can mask the true nature of a lesion, hence, making it difficult to diagnose using only visual examination (ICDAS-II). The DDPen uses fluorescence which makes the diagnosis of a lesion effortless; however, it can also lead to an under-or overestimation. Another explanation is the subjective nature of ICDAS-II, which is not so in the case of DDPen, which uses a pinpoint location to quantify the extent of a lesion. Another notable finding is that the most significant difficulty in diagnosing carious lesions was observed in deeper carious lesions with the lowest accuracy. A possible explanation is the fewer samples in the D3 level. Different cut-off values can be tested to increase the specificity of DDPen at the dentine level. Lussi et al. [33] also found lower specificity of DDPen at dentine level; however, no explanation of this phenomenon was hypothesised. The depth of a carious lesion might influence the absorbance of the laser, influencing the fluorescence of deeper carious lesions.

A change in either $\mathrm{pH}$ or the presence of free radical ions can create a desired anticariogenic environment, as previously noted in the literature $[48,49]$. Another explanation is the limitation of assessing the effect of GC Tooth Mousse Plus ${ }^{\circledR}$ just by focusing on remineralisation. Other assessable properties include surface microhardness, $\mathrm{pH}$ cycling model, and salivary chemical analysis. These properties might reveal a benefit of using GC Tooth Mousse Plus ${ }^{\circledR}$ over conventional fluoride varnish. Although the current study did not find a 


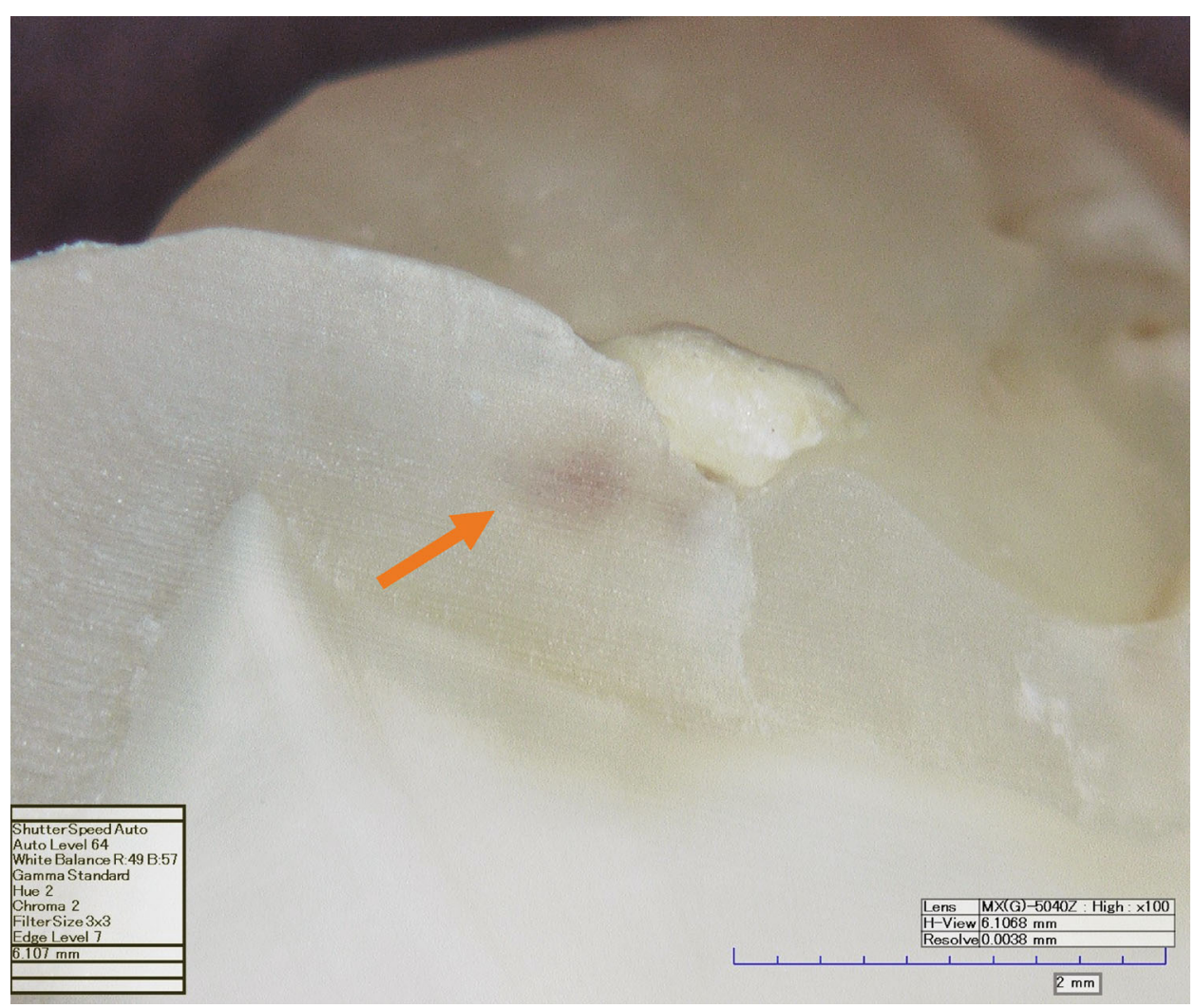

(a)

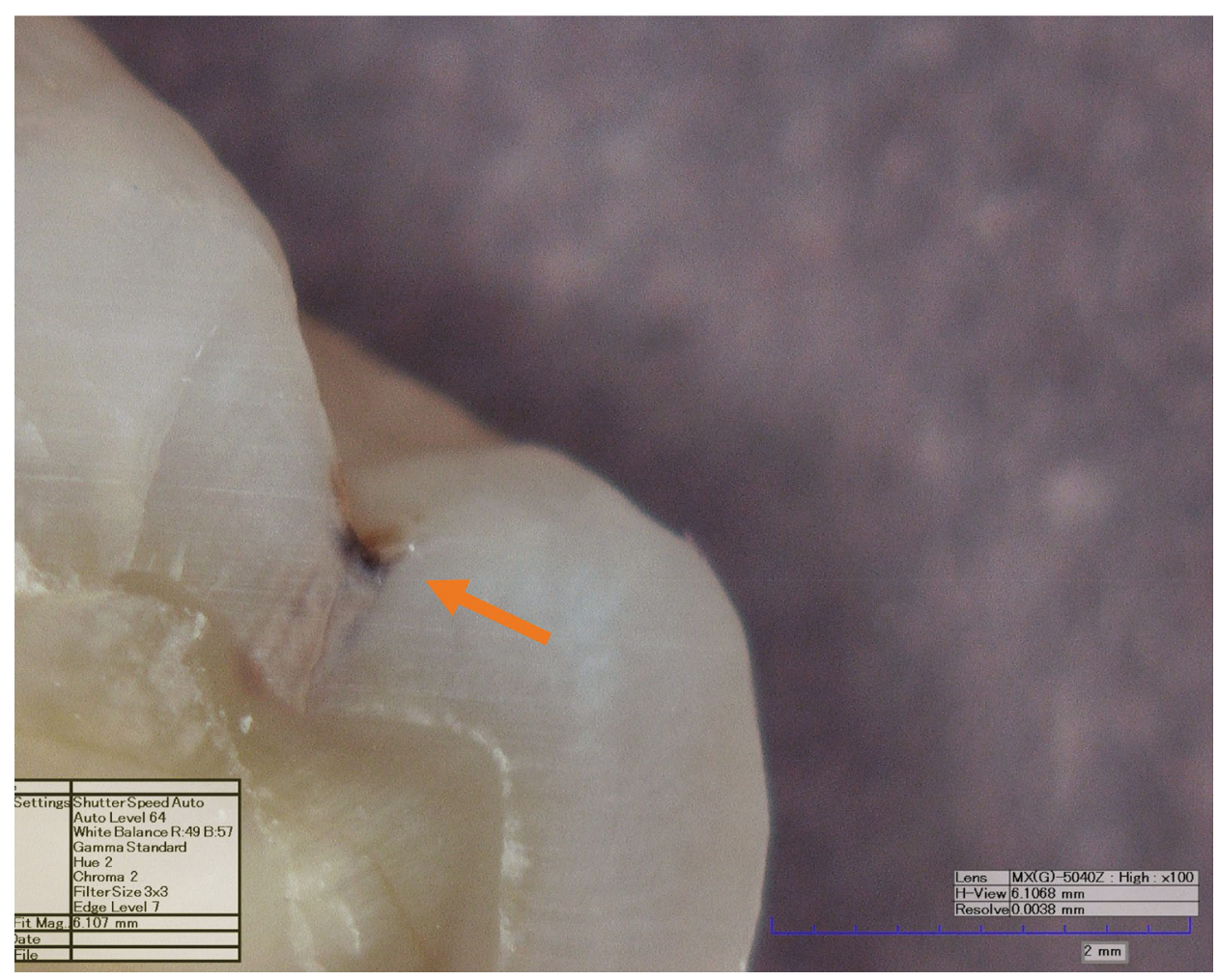

(b)

FIgURe 2: Continued. 


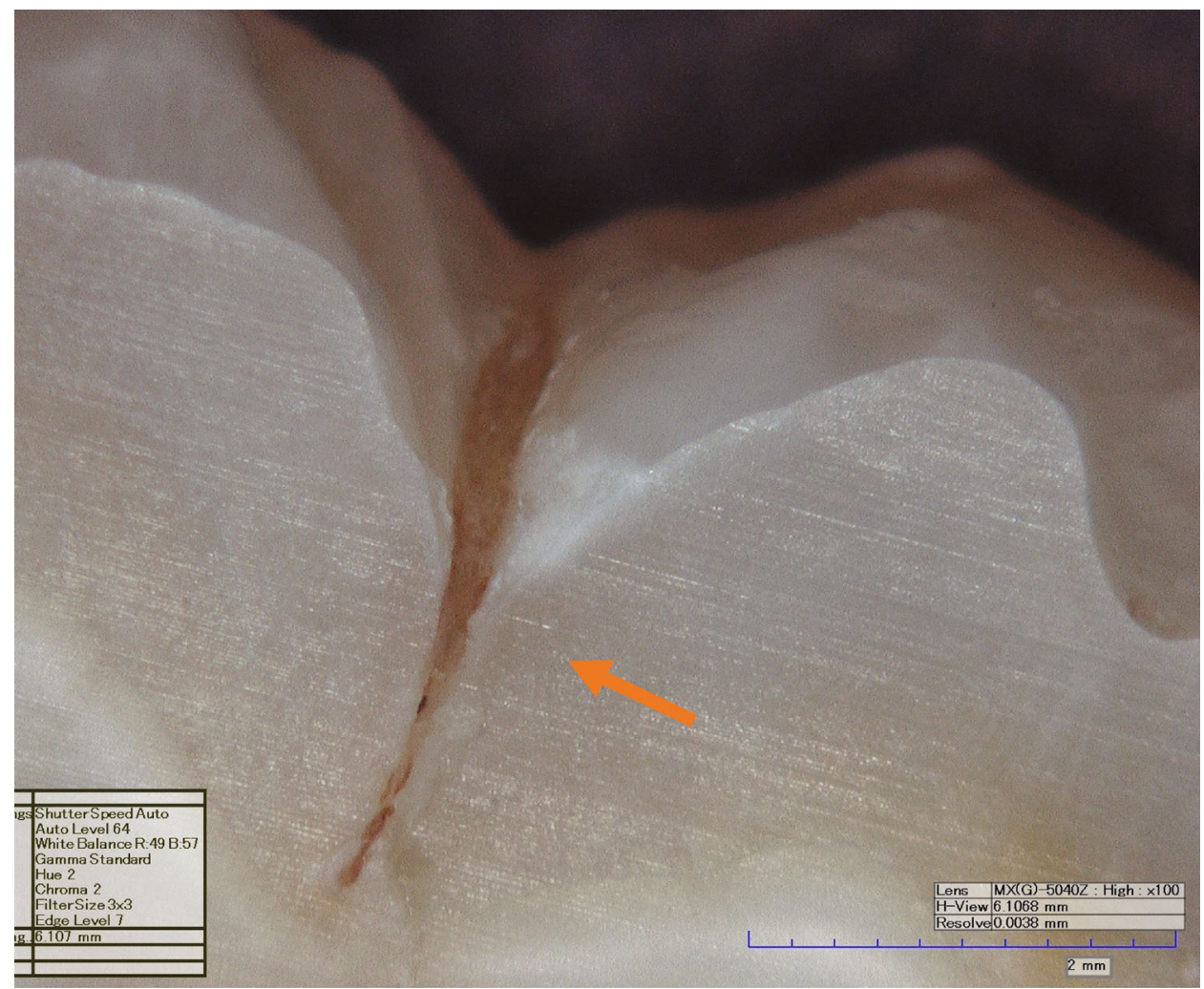

(c)

FIgure 2: Histological section from Hirox digital microscope (KH-7700) (100x magnification) of tooth showing lesion at Downer's classification level (a) D1, (b) D2, and (c) D3.

significant difference between GC Tooth Mousse Plus ${ }^{\circledR}$ and Duraphat ${ }^{\circledR}$ regarding the remineralisation of cavitated carious lesion, one of the CPP-ACP advantages is an extended periodic release of calcium and phosphate ions which pose an anticariogenic effect. However, the current study design did not consider the role of free radical ions or the long-term effect of CPP-ACP over a three- or six-month period, which has been explored previously in the literature [50]. Future studies can focus on a clinical study design that is relatable to reallife clinical practice. The parallel comparison of other diagnostic devices and the currently employed options would allow better comparability of sensitivity and specificity of diagnostic device options. Salivary constituents of pre- and post application of remineralising agents over more extended follow-up periods might reveal exciting findings that can contribute towards the prevention of the carious process.

\section{Conclusion}

The ICDAS-II scoring system and DDPen scoring were able to detect remineralisation. A cut-off value of 0-13 denotes sound tooth, 14-20 denotes enamel caries, and $>21$ denotes dentine caries. The DDPen scoring had a higher-ranked correlation with Downer's histological scoring as compared to ICDAS-II scoring. The area under the ROC curve revealed that DDPen scoring demonstrated higher sensitivity while ICDAS-II scoring demonstrated greater specificity to detect remineralisation.

\section{Data Availability}

The data set used in the current study will be made available at the reasonable request.

\section{Conflicts of Interest}

The authors declare that they have no conflicts of interest to report regarding the present study.

\section{Acknowledgments}

The authors thank Mr. Nuh Mohamad Bin Mohd Makhatar (science officer), School of Dental Sciences, for the technical support with digital microscope images. Support from Universiti Sains Malaysia, short-term grant (304/PPSG/ 6315195) and management of Hospital Universiti Sains Malaysia, Kubang Kerian, Kelantan, in the process of conducting this research, is acknowledged.

\section{References}

[1] S. Akgul, O. Bala, and I. Yikilgan, "Performance of different methods for detection of incipient occlusal caries lesions: an in vitro study," Photomedicine and Laser Surgery, vol. 36, no. 4, pp. 191-197, 2018.

[2] J. E. Frencken, M. C. Peters, D. J. Manton, S. C. Leal, V. V. Gordan, and E. Eden, "Minimal intervention dentistry for 
managing dental caries-a review: report of a FDI task group," International Dental Journal, vol. 62, no. 5, pp. 223-243, 2012.

[3] J. C. Carvalho and H. D. Mestrinho, "Diagnosing noncavitated lesions in epidemiological studies: practical and scientific considerations," Brazilian Oral Research, vol. 28, pp. 1-7, 2014.

[4] I. Floriano, G. Bonini, R. Matos et al., "How different do visuotactile criteria assess caries lesions activity status on occlusal surfaces?," Oral Diseases, vol. 21, no. 3, pp. 299-307, 2015.

[5] M. Tellez and S. Lim, "ICDAS is widely used as standardized and reliable caries detection criteria, but its reporting varies widely," Journal of Evidence Based Dental Practice, vol. 20, no. 1, article 101409, 2020.

[6] S. N. Mohd Said, M. Ekambaram, and C. K. Yiu, "Effect of different fluoride varnishes on remineralization of artificial enamel carious lesions," International Journal of Paediatric Dentistry, vol. 27, no. 3, pp. 163-173, 2017.

[7] Y. Lee, H. Baek, Y. Choi, S. Jeong, Y. Park, and K. Song, "Comparison of remineralization effect of three topical fluoride regimens on enamel initial carious lesions," Journal of Dentistry, vol. 38, no. 2, pp. 166-171, 2010.

[8] A. Al-Jobair, "The effect of repeated applications of enamel surface treatment on in-vitro bovine enamel hardness after multiple exposures to cola drink," Pakistan Oral \& Dental Journal, vol. 30, no. 1, 2010.

[9] K. Venkatesan and M. Ranjan, "Remineralizing agents in dentistry: a review," IOSR Journal of Dental and Medical Sciences, vol. 13, pp. 57-60, 2014.

[10] C. González-Cabezas, H. Jiang, M. Fontana, and G. Eckert, "Effect of low $\mathrm{pH}$ on surface rehardening efficacy of high concentration fluoride treatments on non-cavitated lesions," Journal of Dentistry, vol. 40, no. 6, pp. 522-526, 2012.

[11] R. Rose, "Binding characteristics of Streptococcus mutans for calcium and casein phosphopeptide," Caries Research, vol. 34, no. 5, pp. 427-431, 2000.

[12] R. Rose, "Effects of an anticariogenic casein phosphopeptide on calcium diffusion in streptococcal model dental plaques," Archives of Oral Biology, vol. 45, no. 7, pp. 569-575, 2000.

[13] M. Morgan, G. Adams, D. Bailey, C. Tsao, S. Fischman, and E. Reynolds, "The anticariogenic effect of sugar-free gum containing CPP-ACP nanocomplexes on approximal caries determined using digital bitewing radiography," Caries Research, vol. 42, no. 3, pp. 171-184, 2008.

[14] A. Andersson, K. Sköld-Larsson, A. Haligren, L. G. Petersson, S. Twetman, and A. Hallgren, "Effect of a dental cream containing amorphous cream phosphate complexes on white spot lesion regression assessed by laser fluorescence," Oral health \& preventive dentistry, vol. 5, no. 3, 2007.

[15] N. Cochrane and E. Reynolds, "Calcium phosphopeptides-mechanisms of action and evidence for clinical efficacy," Advances in Dental Research, vol. 24, no. 2, pp. 41-47, 2012.

[16] K. R. Ekstrand, T. Gimenez, F. R. Ferreira, F. M. Mendes, and M. M. Braga, "The international caries detection and assessment system-ICDAS: a systematic review," Caries Research, vol. 52, no. 5, pp. 406-419, 2018.

[17] S. H. Abrams, K. S. Sivagurunathan, J. D. Silvertown et al., "Correlation with caries lesion depth of the canary system, DIAGNOdent and ICDAS II," The Open Dentistry Journal, vol. 11, no. 1, 2017.

[18] N. Patil, S. Choudhari, S. Kulkarni, and S. R. Joshi, "Comparative evaluation of remineralizing potential of three agents on artificially demineralized human enamel: an in vitro study," Journal of conservative dentistry: JCD, vol. 16, no. 2, p. 116, 2013.

[19] I. Zaidi, R. Somani, S. Jaidka, M. Nishad, S. Singh, and D. Tomar, "Evaluation of different diagnostic modalities for diagnosis of dental caries: an in vivo study," International journal of clinical pediatric dentistry, vol. 9, no. 4, p. 320, 2016.

[20] E.-E. Achilleos, C. Rahiotis, A. Kakaboura, and G. Vougiouklakis, "Evaluation of a new fluorescence-based device in the detection of incipient occlusal caries lesions," Lasers in Medical Science, vol. 28, no. 1, pp. 193-201, 2013.

[21] X. Shi, S. Tranaeus, and B. Angmar-Mansson, "Comparison of QLF and DIAGNOdent for quantification of smooth surface caries," Caries Research, vol. 35, no. 1, p. 21, 2001.

[22] X.-Q. Shi, S. Tranæus, and B. Angmar-Månsson, "Validation of DIAGNOdent for quantification of smooth-surface caries: an in vitro study," Acta Odontologica Scandinavica, vol. 59, no. 2, pp. 74-78, 2001.

[23] A. Lussi, R. Hibst, and R. Paulus, "DIAGNOdent: an optical method for caries detection," Journal of Dental Research, vol. 83, pp. 80-83, 2004.

[24] D. Ricketts, "The eyes have it," Evidence-Based Dentistry, vol. 6, no. 3, p. 64, 2005.

[25] J. D. Bader and D. A. Shugars, "A systematic review of the performance of a laser fluorescence device for detecting caries," The Journal of the American Dental Association, vol. 135, no. 10, pp. 1413-1426, 2004.

[26] S. Bhat, K. Hegde, M. Habibullah, and V. Bernhardt, "Incipient enamel lesions remineralization using casein phosphopeptide amorphous calcium phosphate cream with and without fluoride: a laser fluorescence study," Journal of Clinical Pediatric Dentistry, vol. 36, no. 4, pp. 253-355, 2012.

[27] C. Cardoso, A. R. F. de Castilho, P. M. A. Salomão, E. Costa, A. Magalhaes, and M. Buzalaf, "Effect of xylitol varnishes on remineralization of artificial enamel caries lesions in vitro," Journal of Dentistry, vol. 42, no. 11, pp. 1495-1501, 2014.

[28] A. C. Delbem, F. L. Brighenti, A. E. Vieira, and J. A. Cury, "In vitro comparison of the cariostatic effect between topical application of fluoride gels and fluoride toothpaste," Journal of Applied Oral Science, vol. 12, no. 2, pp. 121-126, 2004.

[29] C. Bruun and H. Givskov, "Formation of CaF2 on sound enamel and in caries-like enamel lesions after different forms of fluoride applications in vitro," Caries Research, vol. 25, no. 2, pp. 96-100, 1991.

[30] M. B. Diniz, J. A. Rodrigues, I. Hug, R. De Cássia Loiola Cordeiro, and A. Lussi, "Reproducibility and accuracy of the ICDAS-II for occlusal caries detection," Community Dentistry and Oral Epidemiology, vol. 37, no. 5, pp. 399-404, 2009.

[31] J. R. Landis and G. G. Koch, "The measurement of observer agreement for categorical data," Journal of Biometrics, vol. 33, no. 1, pp. 159-174, 1977.

[32] A. Lussi, B. Megert, C. Longbottom, E. Reich, and P. Francescut, "Clinical performance of a laser fluorescence device for detection of occlusal caries lesions," European Journal of Oral Sciences, vol. 109, no. 1, pp. 14-19, 2001.

[33] A. Lussi, S. Imwinkelried, N. Pitts, C. Longbottom, and E. Reich, "Performance and reproducibility of a laser fluorescence system for detection of occlusal caries in vitro," Caries Research, vol. 33, no. 4, pp. 261-266, 1999.

[34] X.-Q. Shi, U. Welander, and B. Angmar-Månsson, “Occlusal caries detection with KaVo DIAGNOdent and radiography: 
an in vitro comparison," Caries Research, vol. 34, no. 2, pp. 151-158, 2000.

[35] A. Lussi, C. Longbottom, F. Braig, and E. Reich, "Clinical performance of the laser fluorescence system DIAGNOdent for detection of occlusal caries," Caries research, vol. 33, p. 299, 1999.

[36] E. Verdonschot, E. Bronkhorst, R. Burgersdijk, K. König, M. Schaeken, and G. Truin, "Performance of some diagnostic systems in examinations for small occlusal carious lesions," Caries Research, vol. 26, no. 1, pp. 59-64, 1992.

[37] R. Heinrich-Weltzien, J. Kühnisch, T. Oehme, A. Ziehe, L. Stösser, and F. Garcia-Godoy, "Comparison of different DIAGNOdent cut-off limits for in vivo detection of occlusal caries," Operative Dentistry, vol. 28, no. 6, pp. 672-680, 2003.

[38] P. Francescut and A. Lussi, "Correlation between fissure discoloration, Diagnodent measurements, and caries depth: an in vitro study," Pediatric dentistry, vol. 25, no. 6, 2003.

[39] T. Buchwald and Z. Okulus, "Determination of storage solutions influence on human enamel by Raman spectroscopy," Vibrational Spectroscopy, vol. 96, pp. 118-124, 2018.

[40] A. R. Freitas, F. D. Aznar, A. L. Silva, A. Sales-Peres, and S. H. Sales-Peres, "Assessment of the effects of decontamination and storage methods on the structural integrity of human enamel," Revista de Odontologia da UNESP, vol. 45, pp. 59-64, 2016.

[41] C. E. Metz, "Basic principles of ROC analysis," in Seminars in nuclear medicine, pp. 283-298, Elsevier, 1978.

[42] C. Chu, E. Lo, and D. You, "Clinical diagnosis of fissure caries with conventional and laser-induced fluorescence techniques," Lasers in Medical Science, vol. 25, no. 3, pp. 355-362, 2010.

[43] C. Abalos, M. Herrera, A. Jimenez-Planas, and R. Llamas, "Performance of laser fluorescence for detection of occlusal dentinal caries lesions in permanent molars: an in vivo study with total validation of the sample," Caries Research, vol. 43, no. 2, pp. 137-141, 2009.

[44] M. B. Diniz, J. de Almeida Rodrigues, A. B. De Paula, and R. D. Cordeiro, "In vivo evaluation of laser fluorescence performance using different cut-off limits for occlusal caries detection," Lasers in Medical Science, vol. 24, no. 3, pp. 295-300, 2009.

[45] J. E. Iranzo-Cortés, S. Terzic, and J. M. Almerich-Silla, “Diagnostic validity of ICDAS and DIAGNOdent combined: an in vitro study in pre-cavitated lesions," Lasers in Medical Science, vol. 32, no. 3, pp. 543-548, 2017.

[46] Q. Alomari, M. Qudiemat, M. Khalaf, and Y. Al-Tarakemah, "The effect of combining radiographs and DIAGNOdent with visual examination on detection and treatment decisions of noncavitated occluso-dentinal caries," Operative Dentistry, vol. 40, no. 3, pp. 313-321, 2015.

[47] A. C. Pereira, H. Eggertsson, E. A. Martinez-Mier, F. L. Mialhe, G. J. Eckert, and D. T. Zero, "Validity of caries detection on occlusal surfaces and treatment decisions based on results from multiple caries-detection methods," European Journal of Oral Sciences, vol. 117, no. 1, pp. 51-57, 2009.

[48] S. Yadav, V. Sachdev, M. Malik, and R. Chopra, "Effect of three different compositions of topical fluoride varnishes with and without prior oral prophylaxis on Streptococcus mutans count in biofilm samples of children aged 2-8 years: a randomized controlled trial," Journal of Indian Society of Pedodontics and Preventive Dentistry, vol. 37, no. 3, pp. 286-291, 2019.
[49] A. P. Erdem, E. Sepet, T. Avshalom, V. Gutkin, and D. Steinberg, "Effect of CPP-ACP and APF on Streptococcus mutans biofilm: a laboratory study," American Journal of Dentistry, vol. 24, no. 2, pp. 119-123, 2011.

[50] J. Li, X. Xie, Y. Wang et al., "Long-term remineralizing effect of casein phosphopeptide-amorphous calcium phosphate (CPPACP) on early caries lesions in vivo: a systematic review," Journal of Dentistry, vol. 42, no. 7, pp. 769-777, 2014. 